accuracy is required because the weights of the observations are not sufficiently well known to justify it, and ordinary carbon resistors will usually be satisfactory.

The method was devised for the adjustment of networks of observations of differences of gravity, three of which contained respectively nine, fifteen and fifteen nodes, and the numerical details will appear in a publication which is in preparation (see also refs. 3 and 4 ).

In conclusion, I wish to thank the Department of Scientific and Industrial Research for a grant enabling me to take part in the gravity surveys.

Department of Geodesy and Geophysics,

A. H. Cook

Cambridge.

July 27.

${ }^{1}$ Liebmann, G., Nature, 164, 149 (1949).

"Southwell, R. V., "Relaxation Methods in Engineering Science" (Oxford: At the Clarendon Press).

Cook, A. H., and Thirlaway, H. I. S., Report of the XVIII International Geological Congress (in the press).

- Cook, A. H., Mon. Not. Roy. Astro. Soc., Geophys. Supp. (in the press).

\section{Normalization of Frequency Functions}

A METHOD of dealing with non-normal frequency distributions consists of transforming the original variable in such a manner that the transformed variable has a normal distribution. Early writers on this topic were F. Y. Edgeworth and J. C. Kapteyn. In practice, the original law of distribution is unknown, and from our observations we have to choose the form, and estimate the parameters, of a suitable transformation.

May I direct attention to the following technique, which has been found on occasion to give satisfactory results, as judged by the chi-squared test of goodness of fit, without an undue amount of computational labour.

$x$ is a continuous stochastic variable, with probability density $p(x)$. Its range is divided into $k+1$ class intervals $x_{i}-1<x \leqslant x_{i}, i=1$ to $k+1$, and out of $n$ observations, $f_{i}$ fall within the $i$ th class interval.

Defining $y_{i}=\frac{1}{n} \sum_{1}^{i} f_{i}$ and $\alpha_{i}=\int_{-\infty}^{x_{i}} p(x) d x$, it is found, for $n \rightarrow \infty$, that the $y_{i}$, for $i=1$ to $k$, are normally correlated with true means $\alpha_{i}$ and covariances $\alpha_{i}\left(1-\alpha_{i}\right) / n$, where $j \geqslant i$.

Then, if $y_{i}=\int_{-\infty}^{z_{i}} \varphi(t) d t$ and $\alpha_{i}=\int_{-\infty}^{\beta_{i}} \varphi(t) d t$, where $\varphi(t)=\frac{1}{\sqrt{2 \pi}} e^{-t^{2} / 2}$, it is found, for $n \rightarrow \infty$, that the $z_{i}$ are normally correlated with true means $\beta_{i}$ and covariances $\frac{\alpha_{i}\left(1-\alpha_{j}\right)}{n \varphi\left(\beta_{i}\right) \varphi\left(\beta_{j}\right)}$, where $j \geqslant i$ and $i=1$ to $k$.

The 'observed' points $\left(x_{i}, z_{i}\right)$ lie near the curve of the means, $z=f(x)$ say, which contains the points $\left(x_{i}, \beta_{i}\right) . \quad z=f(x)$ is the transformation sought ${ }^{1}$, since $z$ is normally distributed. It remains to choose a suitable form for $f(x)$ and to estimate its parameters. A simple assumption is $f(x)=\sum_{1}^{l} \gamma_{j} x^{j-1}$, where $l<k$. On occasion, $l=4$ or $l=6$ was found to be satisfactory. Then $\beta_{i}=\sum_{1}^{l} \gamma_{j} x_{i}{ }^{j-1}$, and denoting estimates of $\gamma_{j}$ by $\hat{\gamma}_{j}$, these were taken to be the solutions of the equations $X^{\prime} C Z=X^{\prime} C X \hat{\Gamma}^{\prime}$, where $x$ is the matrix $\left\|x_{i}^{j-1}\right\|, C^{-1}$ the matrix $\left\|\frac{\alpha_{i}\left(1-\alpha_{j}\right)}{n \varphi\left(\beta_{i}\right) \varphi\left(\beta_{j}\right)}\right\|, Z^{\prime}$ the vector $\left(z_{i}\right)$ and $\hat{\Gamma}^{\prime}$ is the vector $\left(\hat{\gamma}_{j}\right)$.

These $\hat{\gamma}_{j}$ are, for $n \rightarrow \infty$, normally correlated, with true means $\gamma_{i}$ and coefficient matrix $X^{\prime} C X$. Thus, if the proposed transformation is found to be satis. factory, we then have the correct asymptotic sampling distribution of our estimates of the parameters which appear in it. In computing the $\hat{\gamma}_{j}$, the unknown $\alpha_{i}$ and $\beta_{i}$ were replaced by the $y_{i}$ and the $z_{i}$ respectively. It is considered that for large $n$ the resultant loss of efficiency in the estimates is negligible. The method is useful for psychologists who wish to work from raw scores $x$ to normalized scores $z$ and back again.

\section{J. E. KerRICH}

Sub-Department of Statistics,

University of the Witwatersrand, Johannesburg. June 14.

${ }^{1}$ Cf. Kapteyn, J. C., and Van Uven, M. J., "Skew Frequency Curves in Biology and Statistics" (Groningen, 1916).

\section{Friction and Adhesion of Clean Metals}

IT is now well established that the development of high friction and ultimately of seizure between rubbing metal surfaces is greatly facilitated by progressive cleaning, particularly in the final stages when the last remaining contaminant films of atomic dimensions are removed. We have recently carried out further experiments on the lines of those of Bowden and Hughes ${ }^{1}$, originally with the object of determining the temperature coefficient of friction over a wide temperature range, and have found that with clean surfaces complete seizure occurs.

In our present apparatus, two separate pieces of metal are heated by high-frequency induction to the point of evaporation in a vacuum of about $10^{-6} \mathrm{~mm}$. mercury. They are then allowed to cool nearly to room temperature and are slid together without disturbing the vacuum. The normal load is about $15 \mathrm{gm}$., and the point of contact is localized by one surface being convex. Using pure nickel, we find that complete seizure occurs. Similar results are observed with pure iron and with platinum. There is evidence with iron that, at room temperature, the contaminant film formed even under these conditions can reduce the friction to $\mu=c .3 \cdot 5$. If the metal is heated to $c .300^{\circ} \mathrm{C}$, however, the contamination is sufficiently reduced for complete seizure.

An interesting observation is that under these conditions the sliding process itself leads to a very great increase in the true area of contact. The 'coefficient of friction' $(\mu)$ may rise to 100 or more, as compared with $\mu=6$ in the earlier experiments' ${ }^{1}$. The normal force required to separate the metals has been measured and the area of contact deduced from photomicrographs. It is found that the contact has the bulk strength of the metal, typical figures for iron being $1.18 \mathrm{kgm}$. over $0.0187 \mathrm{~mm} .^{2}$, equivalent to $63 \mathrm{kgm} . / \mathrm{mm} .^{2}$ or 40 tons $/$ in..$^{2}$.

From these results we may conclude that, although our surfaces are probably not absolutely free from an adsorbed monolayer, this residue offers small resistance to the adhesion and recrystallization between 